\title{
Study on Vehicle Rollover Prevention and Ride Comfort Based on AARB
}

\author{
Chen Song ${ }^{1,2, a}$, Xia Changgao ${ }^{1, b}$, Li Shengyong ${ }^{2, c}$, Sun $X^{2, d}$ \\ ${ }^{1}$ School of Automobile and Traffic Engineering, Jiangsu University, Zhenjiang 212013, China \\ ${ }^{2}$ Department of Transportation Engineering, Nantong Shipping College, Nantong 226010, China

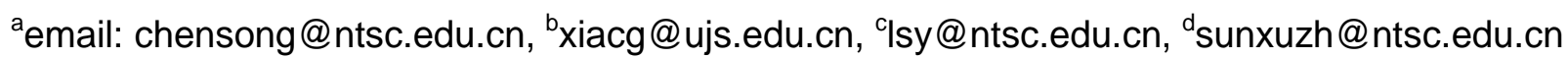

Keywords: AARB; rollover; LQG; Particle Swarm; ride comfort; optimization; dynamic model; simulation;

\begin{abstract}
When interfered by lateral acceleration or uneven road surface, a vehicle is prone to rollover. In this paper, the active anti-roll bar (hereinafter AARB) is self-designed and a six-degree-of-freedom vehicle dynamic model is established for rollover prevention and ride comfort improvement. In order to prevent the rollover and ensure the ride comfort of the vehicle, the linear quadratic (LQ) optimal controller is designed and the weights of the controller are optimized by particle swarm optimization algorithm (PSO). Furthermore, this paper carries out a series of simulation experiments which test the validity of the proposed control strategy in the aspects of body vertical acceleration, roll angle acceleration, roll rate, roll angle, vertical displacement of the suspension and tires, and AARB roll moment. Stimulations show that the method is effective in preventing the rollover and ensuring the ride comfort of a vehicle.
\end{abstract}

\section{Introduction}

Because of vehicle steering or uneven pavement, the traditional roll bar can not adjust roll angle stiffness of suspension in real time. For a vehicle with higher center of gravity, it is prone to causing excessive roll angle of a vehicle body and thus a rollover occurs. In rollover prevention, the following active control technologies----such as semi-active suspension [1], active suspension [2], active steering [3], differential brake [4] and AARB control [5], [6], ----are currently being applied. As the AARB can change the roll angle stiffness of the suspension timely to prevent vehicles from rolling over according to the steering and driving condition of the car, this control technique, compared with several others, can be more direct and effective in rollover prevention. Additionally, AARB has the advantages of low cost and easy application, so it has become a research focus. Many experts have done their research on AARB. Gaffney III et al. introduced an electro-hydraulic controlled AARB with double-loop control system. It was proved to be effective to prevent a rollover of the vehicle with an AARB in simulation and experiment tests [7]. K. JEON et al. utilized an electric control AARB, established a single degree freedom dynamic model, and applied the method of sliding mode control to stop a vehicle rollover [8]. Bal'azs Varga et al. adopted LQ theory to calculate the required roll control torque of the hydraulically controlled AARB for light commercial vehicles [9]. Due to the poor lateral stability of air suspension vehicles, Li Junwei et al. adopted the variable stiffness AARB and gave an angle rigidity relational expression between the front suspension and the rear. The simulation demonstrated that the equipment could stop body roll effectively without influencing the ride comfort [10].

In above literatures, however, only the active stabilizer bar was considered in preventing rollover, and the influence from various parameters of rollover and ride comfort was not taken into comprehensive account. So, there are some limitations. To these shortages, this paper, considering the above influence, designs a LQ optimal controller and applies particle swarm optimization algorithm (PSO) to optimize the weight coefficient of the controller when vehicles disturbed by lateral acceleration and uneven road. 


\section{AARB structure and vehicle dynamic model}

\section{AARB structure}

The schematic diagram of self-designed AARB is shown in Figure1. It is composed of a DC motor, a left harmonic gear mechanism, a right harmonic gear mechanism, a left semi-stabilizer bar, and a right semi-stabilizer bar. Motor armature shaft is connected both to the left wave generator of the harmonic gear and to the right one, so each wave generator is the driving member of the left and the right harmonic gear mechanisms respectively when the motor is rotating. In the left harmonic gear mechanism, the flexible gear is fixed to the shell, and the rigid gear is a follower; in the right harmonic gear mechanism, the rigid gear is fixed to the shell, and the flexible gear is a follower. So the transmission ratio of the left side of the harmonic gear is:

$$
i_{h}=\frac{z_{4}}{z_{4}-z_{3}}=\frac{z_{4}}{z_{0}}
$$

And the transmission ratio of the right side of the harmonic gear is:

$$
i_{h}^{\prime}=-\frac{z_{3}^{\prime}}{z_{4}^{\prime}-z_{3}^{\prime}}=-\frac{z_{3}^{\prime}}{z_{0}}
$$

z3 is the value of the flexible gear teeth of the left side harmonic gear; $\mathrm{z} 4$ is the value of the rigid gear teeth of the left side harmonic gear;z' 3 is the value of the flexible gear teeth of the right side harmonic gear;z' 4 is the value of the rigid gear teeth of the right side harmonic gear.

In the expressions, $z_{4}=z_{3}+z_{0}, z_{4}^{\prime}=z_{3}^{\prime}+z_{0}$.

$\mathrm{z} 0$ is the difference of the two teeth values between the flexible gear and the rigid gear of the harmonic gear mechanisms in both sides.

In order to make the value of the output torque of left semi-stabilizer bar be equal to that of the right and make the direction of the output torque of left semi-stabilizer bar be opposite to that of the right, select $z_{3}^{\prime}=z_{4}$, then $i_{h}=-i_{h}^{\prime}$.

When a vehicle is interfered by lateral acceleration or uneven road surface, the controller controls the motor output torque to make the left and the right semi-stabilizer bar generate an output torque which varies with the body roll angle, the roll rate, and the suspension stroke, etc. The output torque generated by semi-stabilizer bars can reduce the rolling suspension stroke and the body acceleration of a vehicle. So, the driving safety and the ride comfort of the vehicle are greatly improved.

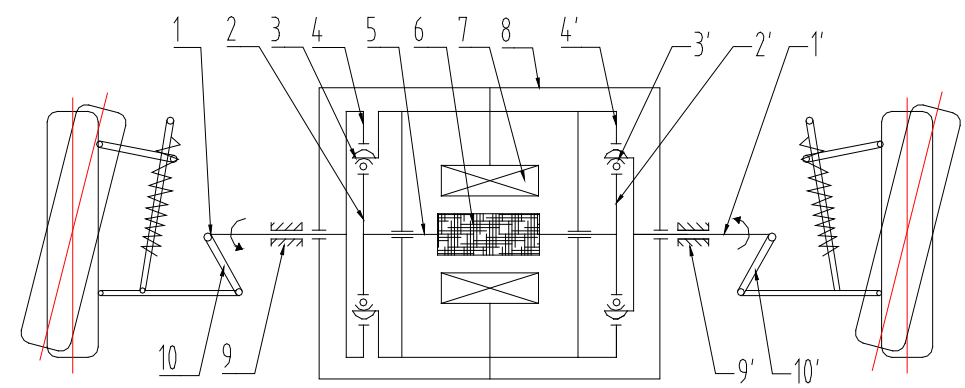

Fig.1 The Schematic diagram of self-designed AARB

1, 1'-left and right semi-stabilizer bar 2-left wave generators 3-left flexible gear 4- left rigid gear 2'- right wave generators 3'-right flexible gear 4'-right rigid gear 5- motor armature shaft 6-motor rotor 7-motor stator 8-shell 9,9'-left and right supports 10, 10'-left and right connecting arm 


\section{Vehicle dynamic model}

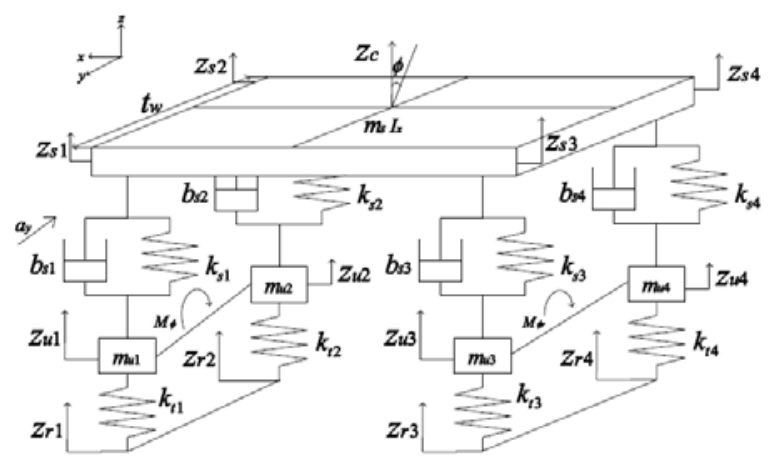

Fig. 2 6-DOF model for vertical and roll motions

As shown in Fig.2, a six-degree-freedom model is established for a vehicle with a front AARB and a rear AARB. This model contains the vertical and the roll motion of the vehicle body, and the vertical motion of the four wheels. The symbols of Fig.1 are found in Table1.

Table1.The symbols of Fig.1

\begin{tabular}{ll}
\hline ms & sprung mass \\
Ix & roll moment of inertia of sprung mass \\
zsi $(\mathrm{i}=1,2,3,4)$ & the vertical displacement of sprung mass \\
$\mathrm{ksi}(\mathrm{i}=1,2,3,4)$ & equivalent stiffness of the suspension \\
$\mathrm{bsi}(\mathrm{i}=1,2,3,4)$ & equivalent damping of the suspension \\
$\mathrm{kti}(\mathrm{i}=1,2,3,4)$ & tire dynamic stiffness \\
mui $(\mathrm{i}=1,2,3,4)$ & the unsprung mass \\
$\mathrm{zri}(\mathrm{i}=1,2,3,4)$ & pavement input corresponding to each wheel \\
$\mathrm{zc}$ & vertical displacement of body centroid \\
$\Phi$ & sprung mass roll angle \\
tw & the vehicle width \\
ay & lateral acceleration \\
\hline
\end{tabular}

The vehicle equipped with two AARB systems in both the front and the rear suspension. In order to maintain the constant steering characteristics of the vehicle, the ratio of the torque generated by the front AARB and the rear is a constant value $\alpha[10]$, which can be obtained as follows:

$$
M_{\phi r}=\alpha M_{\phi f}
$$

In the vehicle model, the disturbances acting on the vehicle are the four road inputs Zr1, Zr2, Zr3, $\mathrm{Zr} 4$ and the lateral acceleration ay. And the control input is a roll moment generated by the AARBs acting on the axle. Then the suspension forces coming from the roll moment are calculated by the following equations:

$$
f_{c f}=\frac{2 M_{\phi f}}{t_{w}}, f_{c r}=\frac{2 M_{\phi r}}{t_{w}}
$$

The suspension forces are as follows:

$$
\begin{aligned}
& f_{1}=-k_{s_{1}}\left(z_{s 1}-z_{u 1}\right)-b_{s_{1}}\left(\dot{z}_{s 1}-\dot{z}_{u 1}\right)-f_{c f} \\
& f_{2}=-k_{s_{2}}\left(z_{s 2}-z_{u 2}\right)-b_{s_{2}}\left(\dot{z}_{s 2}-\dot{z}_{u 2}\right)+f_{c f} \\
& f_{3}=-k_{s_{3}}\left(z_{s 3}-z_{u 3}\right)-b_{s_{3}}\left(\dot{z}_{s 3}-\dot{z}_{u 3}\right)+f_{c r} \\
& f_{4}=-k_{s_{4}}\left(z_{s 4}-z_{u 4}\right)-b_{s_{4}}\left(\dot{z}_{s 4}-\dot{z}_{u 4}\right)-f_{c r}
\end{aligned}
$$

The motion equations for the model of Fig. 1 can be acquired as follows:

$$
I_{x} \ddot{\phi}=\frac{t_{w}}{2}\left(-f_{1}+f_{2}+f_{3}-f_{4}\right)+m_{s} h_{s} a_{y}
$$




$$
\begin{aligned}
& m_{s} \ddot{z}_{c}=f_{1}+f_{2}+f_{3}+f_{4} \\
& m_{1} \ddot{z}_{u 1}=-f_{1}-k_{t 1}\left(z_{u 1}-z_{r 1}\right) \\
& m_{2} \ddot{z}_{u 2}=-f_{2}-k_{t 2}\left(z_{u 2}-z_{r 2}\right) \\
& m_{3} \ddot{z}_{u 3}=-f_{3}-k_{t 3}\left(z_{u 3}-z_{r 3}\right) \\
& m_{4} \ddot{z}_{u 4}=-f_{4}-k_{t 4}\left(z_{u 4}-z_{r 4}\right)
\end{aligned}
$$

The vertical displacements $Z_{s i}(i=1,2,3,4)$ of the left and the right sides of sprung mass can be obtained as follows:

$$
\begin{aligned}
& Z_{s 1}=Z_{c}-\frac{t_{w}}{2} \sin \phi \\
& Z_{s 2}=Z_{c}+\frac{t_{w}}{2} \sin \phi \\
& Z_{s 3}=Z_{c}+\frac{t_{w}}{2} \sin \phi \\
& Z_{s 4}=Z_{c}-\frac{t_{w}}{2} \sin \phi
\end{aligned}
$$

Since the roll angle is small, the $\sin \phi \approx \phi$. Hence, the vertical displacements can be described as follows:

$$
\begin{aligned}
& {\left[\begin{array}{l}
z_{S 1} \\
z_{S 2} \\
z_{S 3} \\
z_{S 4}
\end{array}\right]=\left[\begin{array}{cc}
1 & -\frac{t_{w}}{2} \\
1 & \frac{t_{w}}{2} \\
1 & \frac{t_{w}}{2} \\
1 & -\frac{t_{w}}{2}
\end{array}\right] \cdot\left[\begin{array}{c}
z_{c} \\
\phi
\end{array}\right] \Rightarrow z_{s}=G^{T} \cdot p} \\
& z_{s}=\left[\begin{array}{c}
z_{S 1} \\
z_{S 2} \\
z_{S 3} \\
z_{S 4}
\end{array}\right], G=\left[\begin{array}{cccc}
1 & 1 & 1 & 1 \\
-\frac{t_{w}}{2} & \frac{t_{w}}{2} & \frac{t_{w}}{2} & -\frac{t_{w}}{2}
\end{array}\right], p=\left[\begin{array}{c}
z_{c} \\
\phi
\end{array}\right]
\end{aligned}
$$

With equation (10), the equations of motion are summarized as follows:

$$
\begin{aligned}
& M_{s} \ddot{P}=-G k_{s}\left(G^{T} P-Z_{u}\right)-G B_{s}\left(G^{T} \dot{P}-\dot{Z}_{u}\right)+G F u+L a_{y} \\
& M_{u} \ddot{Z}_{u}=k_{s}\left(G^{T} P-Z_{u}\right)+B_{s}\left(G^{T} \dot{P}-\dot{Z}_{u}\right)-k_{t} Z_{u}-F \cdot u+k_{t} Z_{r}
\end{aligned}
$$

Where

$$
\boldsymbol{K}_{s}=\operatorname{diag}\left(k_{s 1}, k_{s 2}, k_{s 3}, k_{s 4}\right)
$$$$
\boldsymbol{B}_{s}=\operatorname{diag}\left(b_{s 1}, b_{s 2}, b_{s 3}, b_{s 4}\right)
$$$$
\boldsymbol{K}_{t}=\operatorname{diag}\left(k_{t 1}, k_{t 2}, k_{t 3}, k_{t 4}\right)
$$

$\boldsymbol{M}_{s}=\operatorname{diag}\left(m_{s}, I_{x}\right), \boldsymbol{M}_{u}=\operatorname{diag}\left(m_{1}, m_{2}, m_{3}, m_{4}\right), \quad \boldsymbol{L}=\left[\begin{array}{c}0 \\ m_{s} h_{s}\end{array}\right], \quad \mathbf{F}=\frac{2}{t_{w}}\left[\begin{array}{l}-1 \\ 1 \\ \alpha \\ -\alpha\end{array}\right], \quad \mathbf{u}=M_{\varphi f}$

Equation (11) 、 (12) can be rewritten in matrix form as (13).

$$
\begin{aligned}
& {\left[\begin{array}{ll}
\mathbf{M}_{s} & \mathbf{0}_{2 \times 4} \\
\mathbf{0}_{4 \times 2} & \mathbf{M}_{u}
\end{array}\right]\left[\begin{array}{c}
\ddot{\mathbf{p}} \\
\ddot{\mathbf{z}}_{u}
\end{array}\right]=\left[\begin{array}{cc}
-\mathbf{G B}_{s} \mathbf{G}^{T} & \mathbf{G B}_{s} \\
\mathbf{B}_{s} \mathbf{G}^{T} & -\mathbf{B}_{s}
\end{array}\right]\left[\begin{array}{c}
\dot{\mathbf{p}} \\
\dot{\mathbf{z}}_{u}
\end{array}\right]+} \\
& {\left[\begin{array}{cc}
-\mathbf{G K}_{s} \mathbf{G}^{T} & \mathbf{G K}_{s} \\
\mathbf{K}_{s} \mathbf{G}^{T} & -\mathbf{K}_{s}-\mathbf{K}_{t}
\end{array}\right]\left[\begin{array}{c}
\mathbf{p} \\
\mathbf{z}_{u}
\end{array}\right]+\left[\begin{array}{c}
\mathbf{G F} \\
-\mathbf{F}
\end{array}\right] \mathbf{u}+\left[\begin{array}{cc}
\mathbf{0}_{2 \times 4} & \mathbf{L} \\
\mathbf{K}_{t} & \mathbf{0}_{4 \times 1}
\end{array}\right] \mathbf{w}}
\end{aligned}
$$


Where $^{w}=\left[z_{r 1}, z_{r 2}, z_{r 3}, z_{r 4}, a_{y}\right]^{T}$

Equation (11) is transformed into (14).

$\boldsymbol{M}_{e q}\left[\begin{array}{c}\ddot{\boldsymbol{p}} \\ \ddot{\boldsymbol{z}}_{u}\end{array}\right]=\boldsymbol{B}_{e q}\left[\begin{array}{c}\dot{\boldsymbol{p}} \\ \dot{\boldsymbol{z}}_{u}\end{array}\right]+\boldsymbol{K}_{e q}\left[\begin{array}{c}\boldsymbol{p} \\ \mathbf{z}_{u}\end{array}\right]+\boldsymbol{U}_{e q} \boldsymbol{u}+\boldsymbol{L}_{e q} \boldsymbol{w}$

Where

$$
\begin{aligned}
& \boldsymbol{M}_{e q}=\left[\begin{array}{cc}
\boldsymbol{M}_{s} & \boldsymbol{0}_{2 \times 4} \\
\boldsymbol{0}_{4 \times 2} & \boldsymbol{M}_{u}
\end{array}\right], \quad \boldsymbol{B}_{e q}=\left[\begin{array}{cc}
-\boldsymbol{G} \boldsymbol{B}_{s} \boldsymbol{G}^{T} & \boldsymbol{G} \boldsymbol{B}_{s} \\
\boldsymbol{B}_{s} \boldsymbol{G}^{T} & -\boldsymbol{B}_{s}
\end{array}\right], \quad \boldsymbol{K}_{e q}=\left[\begin{array}{cc}
-\boldsymbol{G} \boldsymbol{K}_{s} \boldsymbol{G}^{T} & \boldsymbol{G} \boldsymbol{K}_{s} \\
\boldsymbol{K}_{s} \boldsymbol{G}^{T} & -\boldsymbol{K}_{s}-\boldsymbol{K}_{t}
\end{array}\right], \boldsymbol{U}_{e q}=\left[\begin{array}{c}
\boldsymbol{G} \boldsymbol{F} \\
-\boldsymbol{F}
\end{array}\right], \\
& \boldsymbol{L}_{e q}=\left[\begin{array}{cc}
\boldsymbol{0}_{2 \times 4} & \boldsymbol{L} \\
\boldsymbol{K}_{t} & \boldsymbol{0}_{4 \times 1}
\end{array}\right] .
\end{aligned}
$$

Finally, the state equation for the six-degree-freedom model is shown as (15):

$$
\dot{\boldsymbol{x}}=\boldsymbol{A x}+\boldsymbol{B}_{1} \boldsymbol{w}+\boldsymbol{B}_{2} \boldsymbol{u}
$$

In the equation,

$$
\begin{aligned}
& \boldsymbol{X}=\left[\begin{array}{ll}
\mathbf{z} & \dot{\boldsymbol{z}}
\end{array}\right]^{T}, \quad \mathbf{z}=\left[\begin{array}{llllll}
z_{c} & \phi & Z_{u 1} & z_{u 2} & Z_{u 3} & z_{u 4}
\end{array}\right]^{T}, \quad \boldsymbol{A}=\left[\begin{array}{cc}
\boldsymbol{0}_{6 \times 6} & \boldsymbol{I}_{6 \times 6} \\
\boldsymbol{M}_{e q}^{-1} \boldsymbol{K}_{e q} & \boldsymbol{M}_{e q}^{-1} \boldsymbol{B}_{e q}
\end{array}\right], \quad \boldsymbol{B}_{1}=\left[\begin{array}{c}
\boldsymbol{0}_{6 \times 5} \\
\boldsymbol{M}_{e q}^{-1} \boldsymbol{L}_{e q}
\end{array}\right], \\
& \boldsymbol{B}_{2}=\left[\begin{array}{c}
\boldsymbol{0}_{6 \times 1} \\
\boldsymbol{M}_{e q}^{-1} \boldsymbol{U}_{e q}
\end{array}\right] .
\end{aligned}
$$

\section{Controller design with LQ PSO}

\section{The LQ optimal controller}

Under the disturbances of uneven road and lateral acceleration, for rollover prevention, it is essential to reduce the roll angle, the roll rate, the suspension stroke and tire deflection; for ride comfort, it is necessary to decrease the vertical and the roll accelerations; for energy consumption of active stabilizer bar, it is required to lessen the AARB moment. For these purposes, The LQ objective function is given as follows:

$$
J=\int_{0}^{\infty}\left\{\begin{array}{l}
\rho_{1} \ddot{z}_{c}^{2}+\rho_{2} \ddot{\phi}^{2}+\rho_{3} \phi^{2}+\rho_{4} \dot{\phi}^{2}+\rho_{5}\left(z_{s 1}-z_{u 1}\right)^{2} \\
+\rho_{5}\left(z_{s 2}-z_{u 2}\right)^{2}+\rho_{5}\left(z_{s 3}-z_{u 3}\right)^{2}+\rho_{5}\left(z_{s 4}-z_{u 4}\right)^{2} \\
+\rho_{6} z_{u 1}^{2}+\rho_{6} z_{u 2}^{2}+\rho_{6} z_{u 3}^{2}+\rho_{6} z_{u 4}^{2}+\rho_{7} u^{2}
\end{array}\right\} d t
$$

The output equation is

$$
y=C x+D_{1} w+D_{2} u
$$

Where

$$
\begin{aligned}
& \mathbf{y}=\left[\ddot{z}, \ddot{\varphi}, \varphi, \dot{\varphi}, z_{s 1}-z_{u 1}, z_{s 2}-z_{u 2}, z_{s 3}-z_{u 3}, z_{s 4}-z_{u 4}, z_{u 1}, z_{u 2}, z_{u 3}, z_{u 4}, u\right]^{T} \\
& \boldsymbol{C}=\left[\begin{array}{cccc}
-\boldsymbol{M}_{s}^{-1} \boldsymbol{G} \boldsymbol{K}_{s} \boldsymbol{G}^{T} & \boldsymbol{M}_{s}^{-1} \boldsymbol{G} \boldsymbol{K}_{s} & -\boldsymbol{M}_{s}^{-1} \boldsymbol{G} \boldsymbol{B}_{s} \boldsymbol{G}^{T} & \boldsymbol{M}_{s}^{-1} \boldsymbol{G} \boldsymbol{B}_{s} \\
\boldsymbol{P}_{a} & \boldsymbol{0}_{1 \times 4} & \boldsymbol{0}_{1 \times 2} & \boldsymbol{0}_{1 \times 4} \\
\boldsymbol{0}_{1 \times 2} & \boldsymbol{0}_{1 \times 4} & \boldsymbol{P}_{a} & \boldsymbol{0}_{1 \times 4} \\
\boldsymbol{G}^{T} & -\boldsymbol{I}_{4 \times 4} & \boldsymbol{0}_{4 \times 2} & \boldsymbol{0}_{4 \times 4} \\
\boldsymbol{0}_{4 \times 2} & \boldsymbol{I}_{4 \times 4} & \boldsymbol{0}_{4 \times 2} & \boldsymbol{0}_{4 \times 4}
\end{array}\right] \boldsymbol{D}_{1}=\left[\begin{array}{cc}
\boldsymbol{0}_{2 \times 4} & \boldsymbol{M}_{s}^{-1} \boldsymbol{L} \\
\boldsymbol{0}_{1 \times 4} & 0 \\
\boldsymbol{0}_{1 \times 4} & 0 \\
\boldsymbol{0}_{4 \times 4} & \boldsymbol{0}_{4 \times 1} \\
\boldsymbol{0}_{4 \times 4} & \boldsymbol{0}_{4 \times 1}
\end{array}\right], \quad \boldsymbol{D}_{2}=\left[\begin{array}{c}
\boldsymbol{M}_{s}^{-1} \boldsymbol{G} \boldsymbol{F} \\
0 \\
0 \\
\boldsymbol{0}_{4 \times 1} \\
\boldsymbol{0}_{4 \times 1}
\end{array}\right] .
\end{aligned}
$$

In matrix $\mathrm{C}, \boldsymbol{P}_{a}=\left[\begin{array}{ll}0 & 1\end{array}\right]$.Put the system output equation (17) into the expression (16), the objective function can be written as follows:

$$
J=\frac{1}{2} \int_{0}^{\infty}\left[\boldsymbol{X}^{T} \boldsymbol{Q X}+2 \boldsymbol{X}^{T} \boldsymbol{N U}+\boldsymbol{U}^{T} \boldsymbol{R} \boldsymbol{U}\right] d t
$$

Where $\mathrm{Q}=\mathrm{CTqC}$. $\mathrm{Q}=\mathrm{CTqC}$ is the weight matrix of state variable. $\mathrm{R}=\mathrm{D} 1 \mathrm{Tq} \mathrm{D} 1+\mathrm{r}$ is the weight matrix of controlled inputs. $\mathrm{R}=\mathrm{D} 1 \mathrm{TqD} 1+\mathrm{r}$ and $\mathrm{N}=\mathrm{CTqD} 1$ are the weight matrix of cross-term. $\mathrm{q}=$ 


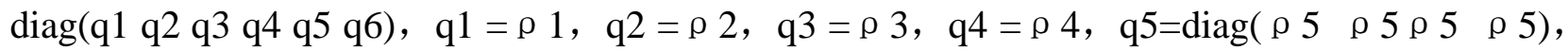

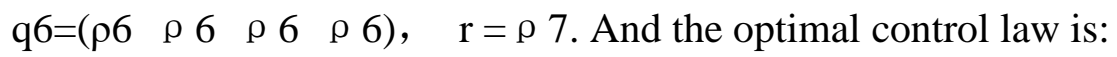

$$
\boldsymbol{U}=-\boldsymbol{K} \boldsymbol{X}
$$

In this paper, the particle swarm optimization algorithm is used to find the optional value of $\mathrm{K}$.

\section{Particle Swarm Optimization Algorithm (PSO) of control parameters}

The selection of weighting matrix $\mathrm{Q} 、 \mathrm{R}$ is the key work of designing an LQ optimal controller [11]. If the LQ optimal controller has fewer weights, the method of trial and error can be applied. And if the LQ optimal controller has more weights, to apply the method of PSO can save time and avoid subjectivity in designing. PSO is a biological evolutionary algorithm [12]. Supposing the position and the velocity of the i-th particle are $\mathrm{xi}=(\mathrm{xi}, 1 \mathrm{xi}, 2 \ldots \mathrm{xi}, \mathrm{d})$ and $\mathrm{vi}=(\mathrm{vi}, 1 \mathrm{vi}, 2 \ldots \mathrm{vi}, \mathrm{d})$ respectively, where the letter $d$ stands for the number of the target optimization variables. The fitness function is expression (16). According to the fitness function, the fitness value of each particle is calculated in the each iteration. Then select the position of the particle whose fitness value is the smallest of each child species as the global optimal solution pg = (pg,1 pg,2 ... pg,d) when initializing. The fitness value of the global optimal solution is recorded as gbest. Suppose the position of each particle is the individual optimal solution, whose fitness value is recorded as pbest. After obtaining the two fitness values, we can adjust each value of the velocity and the position of the particle according to formula (20) and (21).

$$
\begin{aligned}
& v_{i, j}(t+1)=w v_{i, j}(t)+c_{1} r_{1}\left[p_{i, j}-x_{i, j}(t)\right]+c_{2} r_{2}\left[p_{g, j}-x_{i, j}(t)\right] \\
& x_{i, j}(t+1)=x_{i, j}(t)+v_{i, j}(t+1)
\end{aligned}
$$

In above formulas, $\mathrm{j}=1,2, \ldots, \mathrm{d}$; $\mathrm{c} 1$ and $\mathrm{c} 2$ are learning factors; $\mathrm{w}$ is the inertia weight factor; r1and r2 are two random numbers between 0 and 1 .

In order to balance the global search capability and local improvement ability, the inertia weight $\mathrm{w}$ varies automatically with the target fitness value of the particle.

$$
w= \begin{cases}w_{\min }-\frac{\left(w_{\max }-w_{\min }\right) \cdot\left(f-f_{\min }\right)}{\left(f_{\text {vag }}-f_{\min }\right)}, & f \leq f_{\text {avg }} \\ w, & f>f_{\text {avg }}\end{cases}
$$

In above expression, wmax and wmin respectively stand for the maximum and the minimum of w; f denotes current fitness value of particle; favg and fmin respectively represent the current average fitness value and the minimal fitness value of all the particles. Then, the PSO with the optimization objects and the constraints of the controller weights are written in the Matlab program. The operating parameters of particle swarm optimization can be listed as follow: the particle population size is set to 24; the particle size is set to 8 ; assign 100 to the maximum number of iterations; make the learning factor $\mathrm{c} 1=\mathrm{c} 2=2$, the minimum and the maximum inertia weight factor $\mathrm{w}$ are respectively set to wmin $=0.4$ and $\operatorname{wmax}=0.9$; make the maximum particle searching velocity be $20 \%$ of the global particle range; and the search interval of $\rho_{1} 、 \rho_{2} 、 \rho_{3} 、 \rho_{4} 、 \rho_{5}$ 、 $\rho_{6} 、 \rho_{7}$ are set to [1e-2,1e3], [2.5*1e-3,1e3], [1e-2,1e7], [4*1e-4,1e7], [1e1,5*1e7], [1e1,5*1e7], [1e-6,1e-4] respectively. Then, the optimization value $\rho_{1} 、 \rho_{2} 、 \rho_{3} 、 \rho_{4} 、 \rho_{5} 、 \rho_{6} 、 \rho_{7}$ are calculated as130.7076, 740.2098, 2.6985e+05,4.4412e+06,10,10,1.0000e-06 respectively.

\section{Simulations and Results Analysis}

To compare easily, four systems of frequency domain simulations are done. The first is an open loop system without controlling the output parameters of a vehicle. The second is a CASE1 system with emphasizing on the ride comfort. The third is a CASE2 system which pays attention to prevent a rollover. And the last one is an Opt system, which considers the ride comfort and the rollover prevention synthetically. The bode plots of the four systems for road disturbance and lateral acceleration to each output are shown in Fig.3 (a), (b), (c), (d) and Fig.4(a), (b), (c), (d). The 
frequency ranges for the responses under the road disturbance and the lateral acceleration are $1-10 \mathrm{~Hz}$ and $0.5-1 \mathrm{~Hz}$ respectively.

As shown in Fig. 3(a), (b) and (c), compared with the open loop system under a road disturbance input, CASE1、CASE2 and Opt. show improved roll acceleration, smaller roll angle and better roll rate. Meanwhile, it is revealed that among the three systems Opt. is the best in the above three aspects. That means Opt. did the best in improving ride comfort and rollover prevention. However, the results are achieved at the expense of a large suspension stroke in CASE1, CASE2 and Opt. as shown in Fig. 3(d).

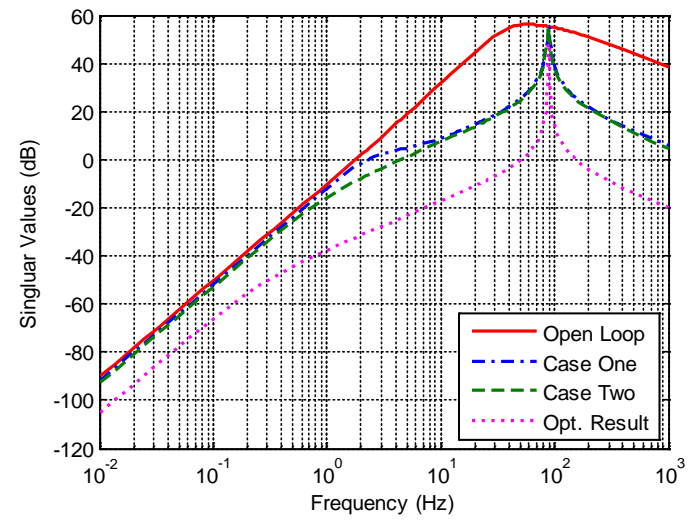

(a) $z_{r} \rightarrow \ddot{\phi}$

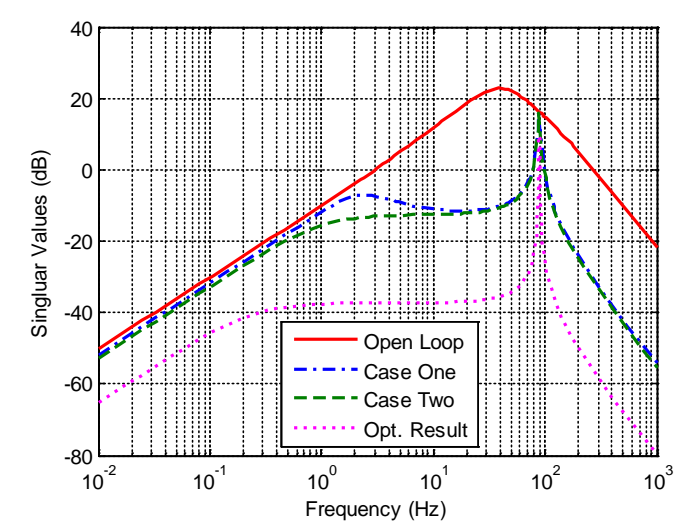

(c) $z_{r} \rightarrow \dot{\phi}$

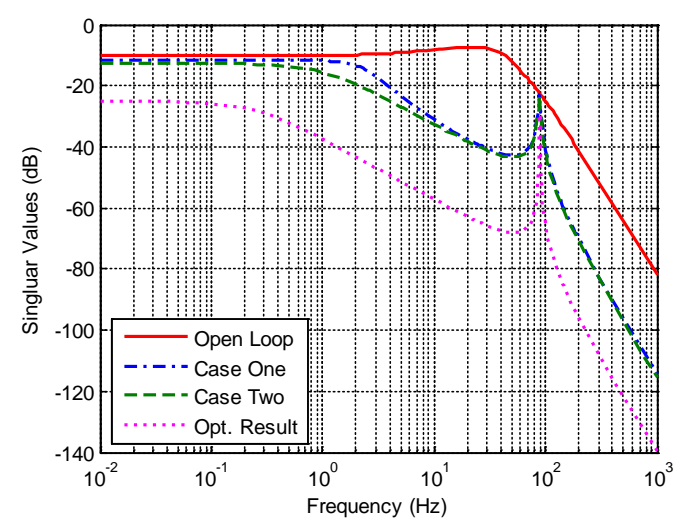

(b) $Z_{r} \rightarrow \phi$

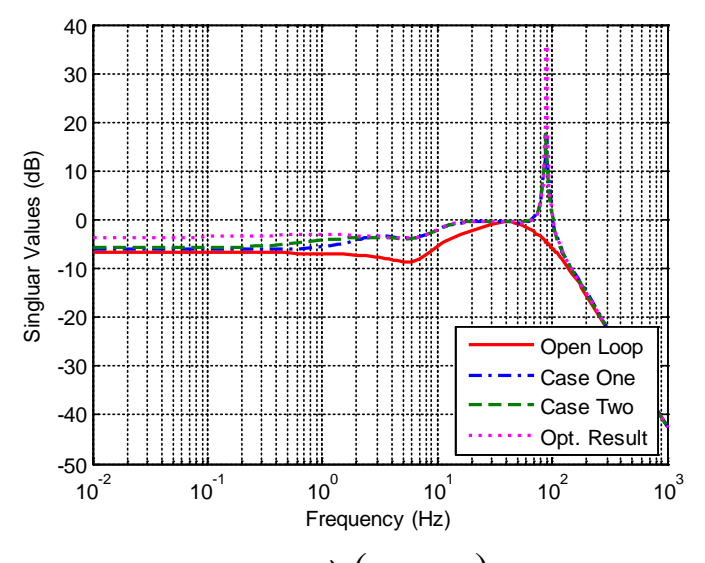

(d) $Z_{r} \rightarrow\left(z_{s}-z_{u}\right)$

Fig.3 Frequency domain simulation under road disturbance

As shown in Fig. 4(a), (b) (c) and (d), compared with the open loop system under lateral acceleration input, CASE2 and Opt. describe improvement in roll acceleration, roll angle, roll rate and suspension stroke. Considering the responses of the roll motion with a road disturbance and a lateral acceleration, it can be concluded that the vehicle with Opt. has the best ride comfort and rollover prevention.

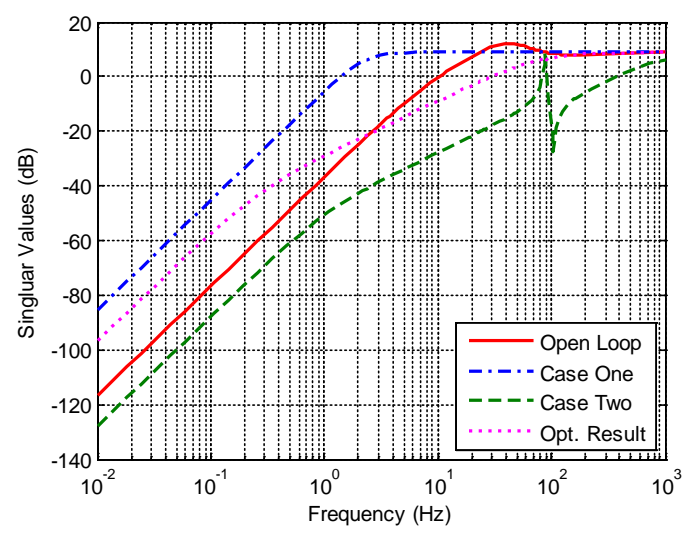

(a) $a_{y} \rightarrow \ddot{\phi}$

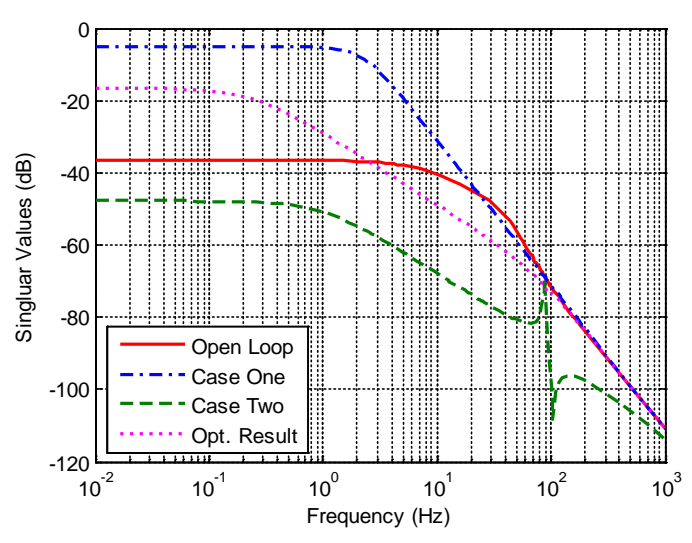

(b) $a_{y} \rightarrow \phi$ 

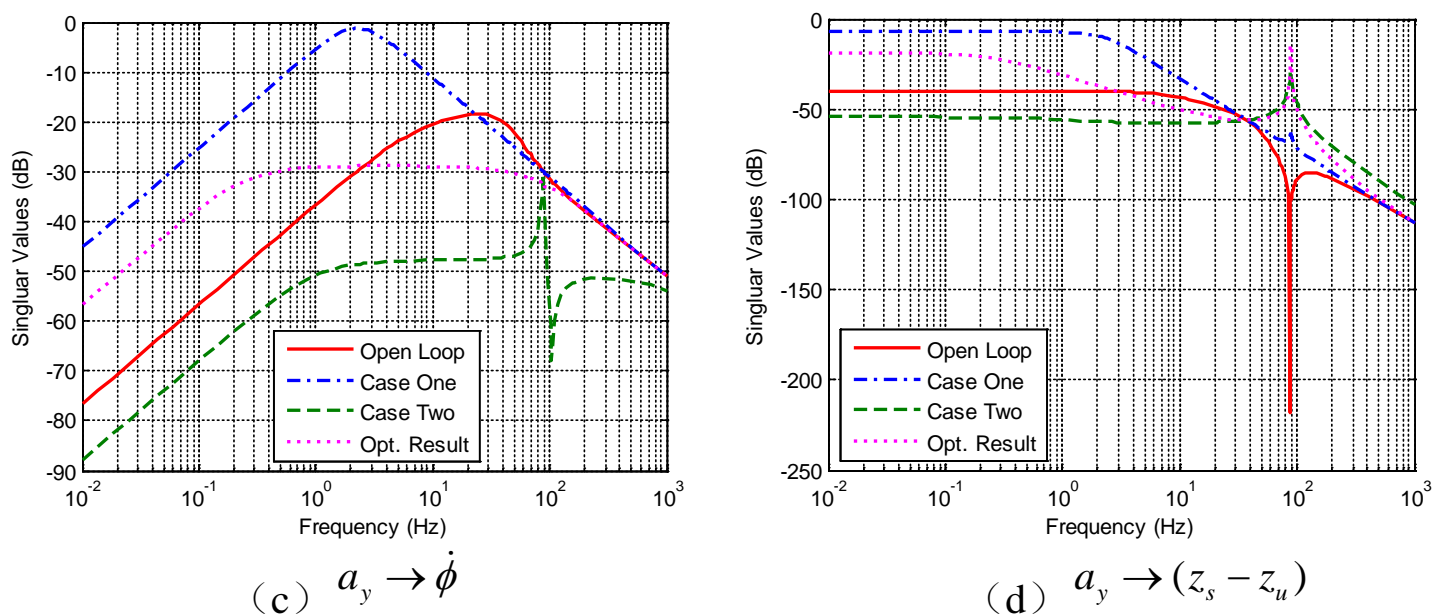

Fig.4Frequency domain simulation under lateral acceleration

To further demonstrate the effect of the AARBs on ride comfort and rollover prevention, two time domain simulations are conducted. The first is a bump simulation, which is performed to validate the ride comfort of the Opt. system. The second is a fishhook maneuver simulation, which is performed to validate the rollover prevention.

The first simulation is carried out when left wheels of the vehicle passes over a bump, defined in (23).

$$
Z_{r}= \begin{cases}\mathrm{c}[1-\cos 20 \pi(t-0.3)] & \text { if } 0.3 \leq t \leq 0.4 \\ 0, & \text { otherwise }\end{cases}
$$

Where, ${ }^{c}=h_{b} / 2$, and $h_{b}$ is the height of the bump.

As shown in Fig.5, Opt. and CASE1 both show a better ride comfort performance than CASE1 and loop open system with a satisfactory attenuation of the effect from road disturbance and roll acceleration. However, as shown in Fig.6, CASE1 demonstrates the worst performance in rollover prevention ---- it may roll over due to its big roll angle and great roll rate. CASE2 and Opt. systems, compared with the open loop system, illustrate better performance of rollover prevention than the other two. So, considering the performance of ride comfort and rollover prevention with the disturbance of uneven road comprehensively, Opt. system is the best choice.

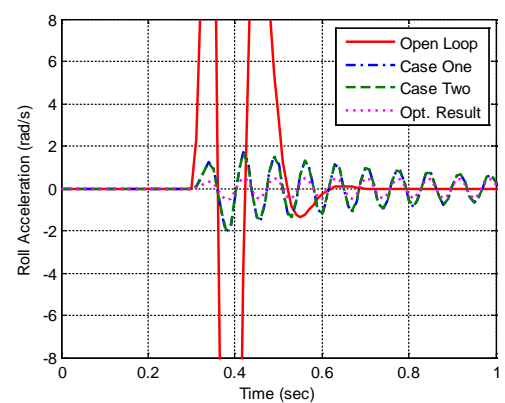

Fig. 5

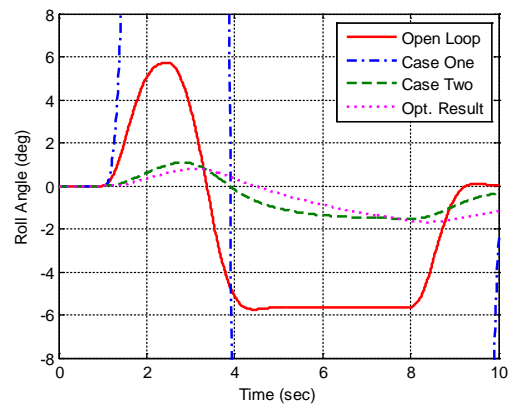

Fig. 6(a)

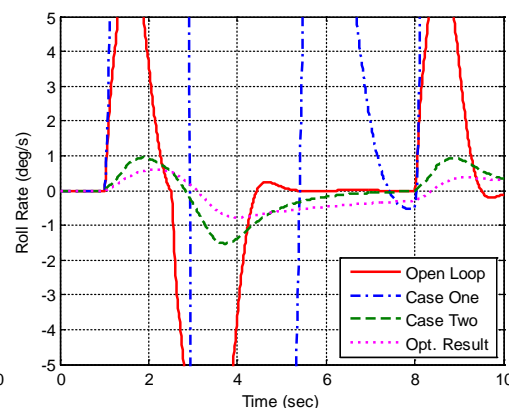

Fig. 6(b)

Fig. 5 the roll accelerations of the bump simulation for each system

Fig. 6(a) the roll rate for each system under fishhook

Fig. 6(b) the roll rate for each system under fishhook

\section{Conclusion}

(1) A six-degree-of-freedom model is established for a vehicle with a self-designed AARB system to study on the rollover prevention and the ride comfort under road disturbance and lateral acceleration. Simultaneously considering the performance of the ride comfort and the rollover prevention, the LQ optimal controller is designed in the model.

(2) The PSO is adopted to optimize the weights of the LQ controller according to the optimization objectives and constraints. 
(3) The simulation of the roll motion under road disturbance and lateral acceleration demonstrates that the capability of vehicles with AARB controlled by LQ Opt. system is superior to that with AARB conducted by other control systems in the ride comfort and the rollover prevention. Therefore, with AARB controlled by LQ Opt. system, the overall performance of the vehicle has been significantly improved.

\section{Acknowledgement}

In this paper, the research was supported by the National Natural Science Foundation of China (grant number 51575001) and Research innovation projects of Jiangsu College graduate (grant no. CXLX12_0629).

\section{References}

[1] Chu Duanfeng, Li Gangyan. A Research on Anti-Rollover Control for Vehicles with Semi-active Suspension [J]. Automotive Engineering, 2012, 5(34): 400-432.

[2] Yim, S,Park, Y. and Yi, K. (2010). Design of active suspension and electronic stability program for rollover prevention. Int. J. Automotive Technology 11, 2, 147-153.

[3] Qiang Li, Guobiao Shi, Yi Lin. Yaw Stability Control of Active Front Steering with Fractional-order PID Cont- roller[C],IEEE Conference ,2009.

[4] Lu J, Messih D, Salib A. An Enhancement to an Electronic Stability Control System to Include a Rollover Control Function [J].SAE, 2007(1): 809-813.

[5] Gosselin-Brisson.S.Bouazara, M., Richard M. J. Design of an active anti-roll bar for off-road vehicles [J] .Shock and Vibration ( S1070-9622),2009 ( 16) : 155-174.

[6] P.H. Cronjé and P.S.Els. Improving off-road vehicle handling using an active anti-roll bar[J].Journal of Terramechanics,vol.47, no.3,pp. 179-189,June 2010.

[7] EF Gaffney III, JC Keane, and JT Nicols, (2009).High efficiency 2 channel active roll control system.SAE Paper No. 2009-01-0222.

[8] K. JEON, H. HWANG, S. CHOI, etc. DEVELOPMENT OF AN ELECTRIC ACTIVE ROLLCONTROL (ARC) ALGORITHM FOR A SUV. International Journal of Automotive Technology, Vol. 13, No. 2, pp. 247-253 (2012).

[9] Bal'azs Varga, Bal'azs N'emeth and P'eter G'asp'ar. Control Design of Anti-Roll Bar Actuator Based on Constrained LQ Method. CINTI 2013.14th IEEE International Symposium on Computational Intelligence and Informatics.19-21November, 2013. Budapest, Hungary 31-36.

[10] Li Junwei,Tang Yingshi, wang weicai, etc. Roll motion control of bus based on variable stiffness anti-roll bar [J],Modern Manufacturing Engineering,2012(8) 12-16.

[11] Guo Yingqing, Xu Zhaodong,Fei Shumin,etal. PSO control used in magnetorheological intelligent stuctures[J]. Journal of vibration and shock, 2011, 30(9): 59-63

[12] Seongjin Yim. Design of a rollover prevention controller with differential game theory and a coevolutionary genetic algorithm. Journal of Mechanical Science and Technology 25 (6) (2011) 1565-1571. 